\title{
BMJ Open Linking medical and dental health record data: a partnership with the Rochester Epidemiology Project
}

\author{
Jennifer L St. Sauver, ${ }^{1,2}$ Alan B Carr, ${ }^{3}$ Barbara P Yawn, ${ }^{1,4}$ Brandon R Grossardt, ${ }^{5}$ \\ Cynthia M Bock-Goodner, ${ }^{6}$ Lori L Klein, ${ }^{1}$ Joshua J Pankratz, ${ }^{6}$ \\ Lila J Finney Rutten, ${ }^{1,2}$ Walter A Rocca ${ }^{1,7}$
}

To cite: St. Sauver JL, Carr AB, Yawn BP, et al. Linking medical and dental health record data: a partnership with the Rochester Epidemiology Project. BMJ Open 2017;7: e012528. doi:10.1136/ bmjopen-2016-012528

- Prepublication history and additional material is available. To view please visit the journal (http://dx.doi.org/ 10.1136/bmjopen-2016012528).

Received 4 May 2016 Revised 19 December 2016 Accepted 21 February 2017

CrossMark

For numbered affiliations see end of article.

Correspondence to Dr Jennifer L St. Sauver; stsauver.jennifer@mayo.edu

\section{ABSTRACT}

Purpose: The purpose of this project was to expand the Rochester Epidemiology Project (REP) medical records linkage infrastructure to include data from oral healthcare providers. The goal of this linkage is to facilitate research studies examining the role of oral health in overall health and quality of life.

Participants: Eight dental practices joined the REP between 2011 and 2015. The REP study team has linked oral healthcare information with medical record information from local healthcare providers for 31750 participants who have resided in Olmsted County, Minnesota. Overall, 17718 (56\%) participants are women, $14318(45 \%)$ are 40 years of age or older and $26090(82 \%)$ are white.

Findings to date: A first study using this new information was recently completed. This resource was used to determine whether the 2007 guidelines from the American Heart Association affected prescription rates of antibiotics to patients with moderate-risk cardiac conditions prior to dental procedures. The REP infrastructure was used to identify a series of patients diagnosed with moderate-risk cardiac conditions by the local healthcare providers ( $n=1351)$, and to abstract antibiotic prescriptions from dental records both pre2007 and post-2007. Antibiotic prescriptions prior to dental procedures declined from $62 \%$ to $7 \%$ following the change in guidelines.

Future plans: Dental data from participating practitioners will be updated on an annual basis, and new dental data will be linked to patient medical records. In addition, we will continue to invite new dental practices to participate in the REP. Finally, we will continue to use this research infrastructure to investigate associations between oral and medical health, and will present findings at conferences and in the scientific literature.

\section{INTRODUCTION}

Oral health is increasingly recognised as a key determinant of overall health and quality of life, and several studies have demonstrated that oral health problems may cause, co-occur with

\section{Strengths and limitations of this study}

- The Rochester Epidemiology Project has linked oral health data from community dental practitioners to electronic health record data from medical providers. This linkage provides a unique opportunity to study associations between oral health and overall health and quality of life.

- This study includes linked data for 31750 participants of all ages and both sexes who have resided in Olmsted County, Minnesota. The sample size makes it possible to study a wide range of oral health questions and outcomes.

- The study does not include all dental providers in Olmsted County, and data are less complete for some segments of the population.

or result from other medical conditions. ${ }^{1-3}$ Oral health problems may lead to a systemic pro-inflammatory state that could contribute to the development of certain medical conditions. For example, persons with chronic periodontitis are at a significantly increased risk of developing cardiovascular diseases compared to persons without this condition, ${ }^{4}$ and poor periodontal health may worsen diabetes. ${ }^{5}$ In addition, the systemic dysregulation of inflammatory responses can cause the concomitant development of oral health problems and other medical conditions. For example, patients with rheumatoid arthritis or dermatomyositis are at high risk of also having oral diseases due to the underlying inflammatory aetiologies of these conditions. ${ }^{6}{ }^{7}$ Conversely, medications used for several chronic conditions, such as asthma, cardiovascular disease, diabetes and osteoporosis, can change the oral environment and contribute to an increased risk of a number of oral health conditions. ${ }^{5-10}$ Despite mounting evidence pointing to the co-occurrence of oral and systemic conditions, research is limited because of the lack of 
comprehensive data resources that link oral health information with systemic health information.

In the USA, dental providers often function as independent, small-business owners, and have not routinely shared data across sites for research purposes. The increasing recognition of oral health as a key contributor to overall health has prompted recent efforts to share oral health data. The most developed of these partnerships is a large, long-standing collaboration between over 30 schools of dentistry called the 'Consortium for Oral Health Research and Informatics (COHRI) '. ${ }^{11}$ This consortium was specifically developed to share electronic oral health data to improve oral health research and education. More recently, six members of COHRI, coordinated by the University of Texas Health Science Center at Houston, have collaborated to share partially de-identified electronic health record data through the 'BigMouth Dental Data Repository'. ${ }^{12}$ Partners are able to query data across all sites to rapidly obtain anonymised data summaries, and more detailed, patient-level data with appropriate approvals. Access to these data is currently limited to partnering institutions, and information about non-oral health is limited to data available in the dental electronic health records with no direct links to patients' medical records. In addition, all of the institutions in this collaboration are based in academic centres and large dental schools. Therefore, the patients cared for at these institutions may not represent the full range of dental problems in the community.

To address these limitations, the Rochester Epidemiology Project (REP), a community collaboration of healthcare providers in southeastern Minnesota and west central Wisconsin, has established partnerships with community dental practitioners in Olmsted County, Minnesota. After obtaining authorisation from patients, dental and medical health data are systematically linked at the individual patient level to better understand the impact of oral health on overall health and health outcomes in the local population.

\section{COHORT DESCRIPTION}

\section{Setting and location}

The REP was established in 1966 by Dr Leonard T. Kurland, as a collaboration between medical care providers in Olmsted County, Minnesota, to share medical record information for research. Each medical care provider in Olmsted County uses a unit (or dossier) medical record system where all data collected for a person are assembled in one place. ${ }^{13}$ The REP links these medical records to unique persons residing in the community, and maintains an electronic index of diagnoses and procedures from these records, including hospitalisations, office visits, emergency room visits, surgical procedures and drug prescriptions. The REP allows investigators to follow subjects across all local medical facilities, regardless of where the care was delivered or of insurance status. Thus, using the resources of the REP, investigators can conduct long-term, population-based studies of disease incidence, prevalence, risk and protective factors, outcomes, health services usage and cost-effectiveness. Over 40 medical care providers have participated in this unique collaboration since 1966, and this resource has been used extensively in research studies that have led to over 2400 scientific publications. ${ }^{13}$ The population of Olmsted County is representative of the population of Minnesota, of the Upper Midwest and of a large segment of the entire US population. ${ }^{14}$ Details regarding the history of the REP and a list of studies that have used the REP are available at the REP website: http://rochesterproject.org/

\section{Recruitment of dental practitioners}

Although a wide range of community healthcare providers have partnered to share their data through the REP, local dental practitioners have not historically participated in this collaboration. However, in July 2008, ABC (Chair of the Department of Dental Specialties at Mayo Clinic) conducted a mailed survey among 96 dentists in over 40 practices in southeastern Minnesota. The survey is included in Appendix A, and was designed to assess the interest of the dentists in different types of research. Overall, $45(47 \%)$ dentists responded to the survey, and 42 indicated they were either 'somewhat' or 'very' interested in research regarding the impact of oral health on general health. With this preliminary information, ABC began an effort in 2009 to develop partnerships with the many interested dental practitioners in the community. He approached the interested dentists through individual meetings, and obtained initial commitments from two dentists in the community to join the REP as new research partners.

Following ABC's initial efforts, the REP team developed specific processes for each new dentist to share data with the REP for research purposes. The first step in each partnership was to establish a data sharing contract and a Health Insurance Portability and Accountability Act (HIPAA) business associate agreement between the dental practitioner and the REP. These agreements explicitly state the rules under which data may be used for research. In particular, the data sharing agreements do not allow the REP, or any investigators that use the REP, to provide details regarding the individual dental practices that participate in the REP (eg, number and types of providers, specific types of services provided, etc). These agreements are in place to ensure that comparisons are not made between practices and that no practice may be singled out as superior or inferior to another practice. Once these agreements were established, dental care providers were then required to obtain Minnesota Research Authorization from their patients. ${ }^{15}$

Minnesota state law requires that healthcare providers obtain authorisation to use health records for research from all patients who received medical care after 1 
January 1997 (Minnesota State privacy law-Statute 144.335, 1997). According to the law, two attempts to obtain this authorisation from each participant must be completed in writing, with at least 60 days between attempts. If the patient gives explicit authorisation, or does not respond after two attempts, then the record can be used for research purposes. Finally, parents must sign the authorisation forms for their children ( $<18$ years), and all children must sign forms for themselves once they become adults ( $\geq 18$ years). All providers that participate in the REP have implemented systems to comply with this law, and authorisation rates are high. In 2000, only $1.7 \%$ of the population refused to allow any of their records to be used for research, and that percentage increased only slightly in 2010 to $2.4 \%$. $^{15}$ This legal requirement represents a significant burden to providers, and remains the single most important barrier to using oral health data for research in our community.

The first community dentist joined the REP in 2011, and implemented the process for obtaining Minnesota Research Authorization beginning in August 2011. Data were first obtained from this practice in March 2013, and were matched to the medical records of persons included in the existing REP research infrastructure. As of May 2015, eight dental practices (30 clinicians) had signed contracts with the REP, collected Minnesota Research Authorization from their patients and began sharing data with the REP. Data from the dental providers are updated annually, and are matched to existing persons in the REP using previously described procedures. ${ }^{16}$ Complete data from all practices are available beginning in 2012 .

\section{Obtaining data from the dental practices}

The REP Administrator (LK) and the REP Programmer (CB) met with dental staff to discuss the type of billing system and the type of dental record used by the practice. The REP team provided a list of desired fields (demographics, billing codes and dates of service) and discussed with office staff the process by which they would record Minnesota Research Authorization status in their systems. All practices chose to record research authorisation as a custom procedure. All participating dental practices used electronic health records, and all captured patient demographic information electronically and billed for their services using American Dental Association (ADA) Current Dental Terminology (CDT) electronic billing codes. However, the type of dental record and billing system varied from site to site. Four sites used Dentrix, three sites used Eaglesoft and one site used a proprietary record. Extraction of the data differed depending on the type of system employed by the practitioner. All data were downloaded at the dental offices by the REP Programmer (CB) on a secure drive, and transported immediately to an REP secure server. Data format differed depending on type of dental record and on the billing system that was used. Data in a comma-separated values (csv) format were simple to work with electronically; however, data were only available in portable document format (pdf) for four sites. These data needed to be extracted via a time-consuming pdf-splitting process before they could be integrated into the REP systems.

\section{Linking dental records to the REP research infrastructure}

All data were standardised prior to incorporation into the REP. Research authorisation status was also extracted from the procedure file and applied to the patient records. ADA categories and CDT code descriptions were applied as part of the standardisation process. Linking medical and dental records was accomplished via the usual REP linkage procedures that have been described previously. ${ }^{16}$ Briefly, patient records were matched electronically via multiple rounds of matching, where the first three rounds of matching were based on a complete match between the records on at least four of the following data points: patient first and last name, date of birth, sex and social security number. Successive rounds of matching used less stringent criteria, including fuzzy matching of name substrings, use of middle initial and Soundex. ${ }^{17}$ Matches of less than four data points relied on address or name history for corroboration. If a match had too few data points for a confirmed match, the data were stored but not incorporated into REP until additional demographic data on the patient are acquired.

Completeness of the demographic information varied from site to site. For example, some sites captured full names, sex, dates of birth and social security numbers, but other sites captured only patient name and address. Missing data were a significant barrier to linkage. However, $\sim 89 \%$ of all dental patients matched to a person in the REP system.

\section{Type and completeness of dental data}

The type and the completeness of the data in the dental records vary substantially across dental practices, and are highly dependent on the needs of the dentist and the individual patient. Some of the records include a detailed medical history and extensive dental details. Other records are brief and limited only to the reason for the visit. Therefore, it is not possible to electronically obtain all of the pertinent details from these records in a standard way that is simple to access for all research studies. However, these limitations are common to medical records as well, and the goal of the REP research infrastructure is to provide complete electronic information when such information is consistently collected and available (eg, demographic data, billing code information, visit dates and location of the dental record). This information then serves as an index to direct a researcher to the areas of the dental record that are most likely to contain the information needed for a particular study. 
For example, if a researcher is interested in conducting a study related to dental implants, the REP staff will provide the researcher with a list of patients with a billing code for dental implants, the date of the service and the name of the practice at which the implant was performed. The REP staff will then arrange a time for the researcher to visit the dental practice and review the dental record to abstract the pieces of information pertinent to the study. All of the dental partners have agreed to facilitate these visits and to provide space for a visiting researcher. Therefore, all information in the dental records is accessible to an interested researcher. However, as the types and amount of data collected differ dramatically from practice to practice, it is often necessary for a researcher to review all possible dental and medical records to collect the relevant information for the study.

\section{Number of patients with dental data}

Using the REP Census, we identified all persons who resided in Olmsted County, Minnesota, at any time from 1 January 2013 through 31 December $2013 .{ }^{16}$ We then identified all persons who received a dental service of any kind between 2012 and 2014. The proportion of persons for whom dental data were available is presented separately by sex, four broad age groups $(0-18$, 19-39, 40-64 and 65+ years), race (white, black, Asian and other race) and ethnicity (Hispanic). $\chi^{2}$ tests were performed to determine whether the availability of dental information differed by these characteristics. In addition, among persons for whom dental data were available, we describe the frequency of various types of dental services, separately across the four age groups.

As of 14 April 2015, 46973 patients have been asked to sign Minnesota Research Authorization for their dental records, and 42745 (91\%) have granted authorisation for use of their dental record for research. The amount of dental data varies across practices, but all practices have shared at least 3 years of data (20122014). Data consist of demographic information (name, sex, date of birth) and dental service data. Dental service data are coded using the ADA and CDT codes, and dates of each service are also available. Between 2012 and 2014, 31750 patients received nearly 477000 dental services. Persons with at least some dental data in the REP are similar to persons who do not yet have dental data in the REP (table 1); however, male patients, patients between the ages of 19-39 years, and non-white patients are less likely to have dental data. The median number and types of services received during this time frame are shown by age group in table 2 . Diagnostic services were the most common service for all age groups; however, other services differed by age. For example, $74 \%$ of children (0-18 years) had at least one preventive service code compared with $50 \%$ of older adults (65+ years). Conversely, older adults $(65+$ years $)$ were more likely to have a removable service code $(47 \%)$ compared with only $2 \%$ of children ( $0-18$ years).

\section{FINDINGS TO DATE}

As an initial proof-of-concept project, a team of Mayo Clinic investigators used this resource to determine whether the 2007 guidelines from the American Heart Association affected prescription rates of antibiotics to patients with moderate-risk cardiac conditions prior to dental procedures. They used the existing REP research infrastructure to identify all patients residing in Olmsted County, Minnesota, between 2005 and 2014 with a cardiac condition that carries a moderate or high risk of developing infective endocarditis. The investigators reviewed the dental records for patients that received dental care with a participating dental practitioner $(\mathrm{n}=1351$ patients with 8787 dental visits). They found that antibiotic prescriptions prior to dental procedures declined from $62 \%$ to $7 \%$ in this group of patients following the change in guidelines. ${ }^{18}$ This study demonstrates the utility of the expanded REP research infrastructure to address research questions that encompass medical and oral health.

\section{Strengths and limitations}

A major strength of this expansion is the availability of linked medical and dental health data for over 30000 individuals. This large sample size provides a unique opportunity to address a wide range of oral health research questions. A limitation of this project is the relatively small number of dental partners participating in the REP. In 2014, more than 40 private dental practices were located in Olmsted County; however, only eight currently participate in the REP. We are not able to compare the type of dental data available for these eight practices to dental data for the entire county, because such data are not available. It is possible that the data available from these eight practices are not representative of the type of dental care received by all community members. In addition, the proportion of persons of black race or Hispanic ethnicity with available dental data is lower than the proportion of persons with available medical data, indicating that we are disproportionately missing dental data on our racial/ethnic minority population. Further efforts to engage other community dental practitioners will provide a more complete picture of the oral health for the entire community. In particular, Olmsted County has a single oral health service which provides free or low-cost dental services to the underinsured and uninsured local population, and discussions are ongoing with this service for inclusion in the REP.

\section{COLLABORATION}

We have successfully expanded a community-based partnership of healthcare providers to include information from eight community dental practitioners for over 30000 persons. Data are linked at the person level, and it is now possible to identify cohorts of patients who received a particular type of dental service and to 
Table 1 Comparison of persons with and without dental data included in the Rochester Epidemiology Project* (data from 2012 to 2014)

\begin{tabular}{|c|c|c|c|c|c|c|}
\hline \multirow[b]{2}{*}{ Characteristic } & \multirow{2}{*}{$\begin{array}{l}\text { Population } \\
\text { Total N }\end{array}$} & \multicolumn{2}{|c|}{ Have dental data } & \multicolumn{2}{|c|}{ Have no dental data } & \multirow{2}{*}{$\begin{array}{l}\mathrm{p} \text { Value for } \\
\text { comparison }\end{array}$} \\
\hline & & $\mathbf{N}$ & Per cent & $\mathbf{N}$ & Per cent & \\
\hline All persons & 158786 & 31750 & 20.0 & 127036 & 80.0 & - \\
\hline \multicolumn{7}{|l|}{ Sex } \\
\hline Men & 75130 & 14032 & 18.7 & 61098 & 81.3 & $<0.0001$ \\
\hline Women & 83656 & 17718 & 21.2 & 65938 & 78.8 & - \\
\hline \multicolumn{7}{|l|}{ Age, years } \\
\hline $0-18$ & 42786 & 9752 & 22.8 & 33034 & 77.2 & $<0.0001$ \\
\hline 19-39 & 47270 & 7680 & 16.2 & 39590 & 83.8 & - \\
\hline $40-64$ & 48281 & 10129 & 21.0 & 38152 & 79.0 & - \\
\hline $65+$ & 20449 & 4189 & 20.5 & 16260 & 79.5 & - \\
\hline \multicolumn{7}{|l|}{ Race } \\
\hline White & 127781 & 27217 & 21.3 & 100564 & 78.7 & $<0.0001$ \\
\hline Black & 9517 & 1021 & 10.7 & 8496 & 89.3 & - \\
\hline Asian & 8686 & 1703 & 19.6 & 6983 & 80.4 & - \\
\hline Other & 12802 & 1809 & 14.1 & 10993 & 85.9 & - \\
\hline \multicolumn{7}{|l|}{ Ethnicity } \\
\hline Hispanic & 10579 & 1794 & 17.0 & 8785 & 83.0 & $<0.0001$ \\
\hline Non-Hispanic & 148207 & 29956 & 20.2 & 118251 & 79.8 & - \\
\hline
\end{tabular}

${ }^{*}$ The population was defined at the 2013 Rochester Epidemiology Project Census.

$+\chi^{2}$ test of homogeneity to determine whether the frequency counts are distributed similarly across persons who do or do not have dental data.

$\ddagger$ The other race category includes persons of a race other than white, black or Asian and persons with unknown race.

Table 2 Distribution of type of service by age for both sexes and all races and ethnicities combined (data from 2012 to 2014)

\begin{tabular}{|c|c|c|c|c|c|c|c|c|}
\hline \multirow[b]{3}{*}{ Type of service } & \multicolumn{8}{|l|}{ Age group } \\
\hline & \multicolumn{2}{|c|}{$0-18$ years $(\mathrm{N}=9752)$} & \multicolumn{2}{|c|}{$19-39$ years $(\mathrm{N}=7680)$} & \multicolumn{2}{|c|}{$40-64$ years $(\mathrm{N}=10129)$} & \multicolumn{2}{|c|}{$65+$ years $(N=4189)$} \\
\hline & $N(\%)^{*}$ & $\begin{array}{l}\text { Med } \\
(Q 1, Q 3) \dagger\end{array}$ & $N(\%)^{*}$ & $\begin{array}{l}\text { Med } \\
(Q 1, \text { Q3) } \dagger\end{array}$ & $N(\%)^{*}$ & $\begin{array}{l}\text { Med } \\
(Q 1, Q 3) \dagger\end{array}$ & $N(\%)^{*}$ & $\begin{array}{l}\text { Med } \\
(Q 1, \text { Q3)† }\end{array}$ \\
\hline Diagnostic & $8397(86.1)$ & $5(3,8)$ & $6521(84.9)$ & $5(3,7)$ & $9080(89.6)$ & $6(3,9)$ & $3648(87.1)$ & $5(3,8)$ \\
\hline Preventive & 7207 (73.9) & $6(3,10)$ & 5344 (69.6) & $3(2,6)$ & $6438(63.6)$ & $4(2,6)$ & 2075 (49.5) & $4(2,6)$ \\
\hline Restorative & $2418(24.8)$ & $2(1,4)$ & 2626 (34.2) & $2(1,4)$ & $4668(46.1)$ & $2(1,4)$ & 1817 (43.4) & $2(1,4)$ \\
\hline Removable & $211(2.2)$ & $1(1,2)$ & 1503 (19.6) & $2(1,3)$ & 3563 (35.2) & $3(1,6)$ & 1977 (47.2) & $5(2,8)$ \\
\hline Adjunctive general & $2080(21.3)$ & $1(1,2)$ & $1581(20.6)$ & $1(1,2)$ & $2887(28.5)$ & $2(1,3)$ & $868(20.7)$ & $2(1,3)$ \\
\hline O \& M surgery $\ddagger$ & $2553(26.2)$ & $1(1,2)$ & $1340(17.4)$ & $1(1,1)$ & 1839 (18.2) & $1(1,2)$ & 1065 (25.4) & $1(1,2)$ \\
\hline Orthodontics & $1132(11.6)$ & $2(1,3)$ & $404(5.3)$ & $1(1,2)$ & $377(3.7)$ & $1(1,3)$ & $38(0.9)$ & $1(1,2)$ \\
\hline Endodontics & $262(2.7)$ & $1(1,2)$ & $334(4.3)$ & $1(1,2)$ & $660(6.5)$ & $1(1,2)$ & 204 (4.9) & $1(1,2)$ \\
\hline Fixed & $2(<0.1)$ & $1(1,1)$ & $20(0.3)$ & $3(1,4)$ & $94(0.9)$ & $2(1,4)$ & $103(2.5)$ & $2(1,4)$ \\
\hline Prosthetics & $6(0.1)$ & $1(1,1)$ & $10(0.1)$ & $1(1,1)$ & $42(0.4)$ & $1(1,1)$ & $18(0.4)$ & $1(1,1)$ \\
\hline Implant services & $15(0.2)$ & $1(1,3)$ & $208(2.7)$ & $1(1,2)$ & $781(7.7)$ & $2(1,3)$ & 391 (9.3) & $2(1,3)$ \\
\hline
\end{tabular}

${ }^{*}$ Among all persons with some dental information, this is the number (and per cent) of persons with a specific dental service.

†Among persons who received a service of this type, this is the median number of services received and the 25th centile (Q1) and 75th centile (Q3).

‡Oral and maxillofacial surgery.

determine whether such services are associated with other types of health outcomes. It is also possible to identify persons with a specific disease or condition and assess the use of dental services either before or after the diagnosis. We welcome inquiries regarding the use of this research infrastructure for specific projects, and have a long history of collaboration. Details regarding access to REP data for research are available on our website at: http://www.rochesterproject.org. For further information, please contact us at info@rochesterproject.org.
Author affiliations

${ }^{1}$ Division of Epidemiology, Department of Health Sciences Research, Mayo Clinic, Rochester, Minnesota, USA

${ }^{2}$ Robert D. and Patricia E. Kern Center for the Science of Health Care Delivery, Mayo Clinic, Rochester, Minnesota, USA

${ }^{3}$ Department of Dental Specialties, Mayo Clinic, Rochester, Minnesota, USA

${ }^{4}$ Department of Research, Olmsted Medical Center, Rochester, Minnesota, USA

${ }^{5}$ Division of Biomedical Statistics and Informatics, Department of Health Sciences Research, Mayo Clinic, Rochester, Minnesota, USA

${ }^{6}$ Department of Information Technology, Mayo Clinic, Rochester, Minnesota, USA

${ }^{7}$ Department of Neurology, Mayo Clinic, Rochester, Minnesota, USA 
Acknowledgements The authors would like to thank our dental partners for their collaboration. The authors would also like to thank Robin Adams for her assistance with manuscript preparation and submission.

Contributors JLSS, ABC, BPY, LJFR and WAR were involved in the conception and design of the study. ABC, CMB-G, LLK and JJP engaged dental practitioners and collected the data. JLSS, BRG, CMB-G and WAR participated in data analysis. All authors contributed to interpretation of the data and critical revision of the manuscript. All authors also approved the final version to be published.

Funding This study was made possible using the resources of the Rochester Epidemiology Project, which is supported by the National Institute on Aging of the National Institutes of Health under award number R01AG034676. This study was also supported by the Robert D. and Patricia E. Kern Mayo Clinic Center for the Science of Healthcare Delivery Population Health Research Program.

Disclaimer The content is solely the responsibility of the authors and does not necessarily represent the official views of the National Institutes of Health or the Center for the Science of Healthcare Delivery.

Competing interests None declared.

Ethics approval This study was approved by the Mayo Clinic and Olmsted Medical Center Institutional Review Boards. Informed consent was waived; however, we did not include data for any patients who had not given permission for their medical or oral health records to be used for research (Minnesota Research Authorization).

Provenance and peer review Not commissioned; externally peer reviewed.

Data sharing statement Researchers can request access to the data by submitting a request to info@rochesterproject.org. The Rochester Epidemiology Project website provides further information for interested investigators: http://rochesterproject.org/

Open Access This is an Open Access article distributed in accordance with the Creative Commons Attribution Non Commercial (CC BY-NC 4.0) license, which permits others to distribute, remix, adapt, build upon this work noncommercially, and license their derivative works on different terms, provided the original work is properly cited and the use is non-commercial. See: http:// creativecommons.org/licenses/by-nc/4.0/

\section{REFERENCES}

1. Razak PA, Richard KM, Thankachan RP, et al. Geriatric oral health: a review article. J Int Oral Health 2014;6:110-16.

2. Dutt $P$, Chaudhary S, Kumar P. Oral health and menopause: a comprehensive review on current knowledge and associated dental management. Ann Med Health Sci Res 2013;3:320-3.
3. Slade GD. Oral health-related quality of life is important for patients, but what about populations? Community Dent Oral Epidemio 2012;40(Suppl 2):39-43.

4. Bahekar AA, Singh S, Saha S, et al. The prevalence and incidence of coronary heart disease is significantly increased in periodontitis: a meta-analysis. Am Heart $J$ 2007;154:830-7.

5. Tavares M, Lindefjeld Calabi KA, San Martin L. Systemic diseases and oral health. Dent Clin North Am 2014;58: 797-814.

6. Zalewska A, Waszkiewicz N, Szajda SD, et al. Impact of salivary flow and lysozyme content and output on the oral health of rheumatoid arthritis patients. Postepy Hig Med Dosw (Online) 2011;65:40-5.

7. Tanaka TI, Geist SM. Dermatomyositis: a contemporary review for oral health care providers. Oral Surg Oral Med Oral Pathol Oral Radiol 2012;114:e1-8.

8. Widmer RP. Oral health of children with respiratory diseases. Paediatr Respir Rev 2010;11:226-32.

9. Lam OL, Zhang W, Samaranayake LP, et al. A systematic review of the effectiveness of oral health promotion activities among patients with cardiovascular disease. Int J Cardiol 2011;151:261-7.

10. Anil S, Preethanath RS, AlMoharib HS, et al. Impact of osteoporosis and its treatment on oral health. Am J Med Sci 2013;346:396-401.

11. Stark PC, Kalenderian E, White JM, et al. Consortium for ora health-related informatics: improving dental research, education, and treatment. J Dent Educ 2010;74:1051-65.

12. Walji MF, Kalenderian E, Stark PC, et al. BigMouth: a multi-institutional dental data repository. J Am Med Inform Assoc 2014;21:1136-40.

13. Rocca WA, Yawn BP, St Sauver JL, et al. History of the Rochester Epidemiology Project: half a century of medical records linkage in a US population. Mayo Clin Proc 2012;87:1202-13.

14. St Sauver JL, Grossardt BR, Leibson CL, et al. Generalizability of epidemiological findings and public health decisions: an illustration from the Rochester Epidemiology Project. Mayo Clin Proc 2012;87:151-60.

15. St Sauver JL, Grossardt BR, Yawn BP, et al. Data resource profile: the Rochester Epidemiology Project (REP) medical records-linkage system. Int J Epidemiol 2012;41:1614-24.

16. St Sauver JL, Grossardt BR, Yawn BP, et al. Use of a medical records linkage system to enumerate a dynamic population over time: the Rochester Epidemiology Project. Am J Epidemiol 2011:173:1059-68.

17. Roesch $A$. Matching data using sounds-like operators and $S A S \otimes$ compare functions. In: SAS, ed. SAS Global Forum 2012, 2012:1-11.

18. DeSimone DC, El Rafei A, Challener DW, et al. Impact of the American Heart Association's 2007 guidelines on the practice of dental prophylaxis for the prevention of infective endocarditis in Olmsted County, MN. Mayo Clin Proc (in press). 